\title{
Pair Formation in Sexually-Transmitted Diseases
}

$\mathrm{BU}-1034-\mathrm{M}$

June, 1989

03 


\title{
PAIR FORMATION IN SEXUALLY-TRANSMITTED DISEASES
}

\author{
Roland Waldstätter \\ Dept. of Biomathematics \\ Auf der Morgenstelle 10 \\ 7400 Tübingen 1 \\ West Germany
}

\begin{abstract}
Most epidemiological models of sexually-transmitted diseases (STD's) consider populations of single individuals. These models assume that every encounter by a susceptible possibly involves a different partner and such individuals get infected, with a constant probability per encounter, by infected partners. In order to match the model with data it is assumed that the probability of infection per "encounter" sums over all sexual contacts during a partnership. Although in reality the majority of individuals live in steady partnerships, it is usually assumed that these models are good approximations.

Models that use a different approach show other results. This paper presents a brief overview of recent models that take into account pair formation and explicitly follow pairs in the equations. The effect of prostitution on the Dietz/Hadeler model is investigated. Some results are compared with those from the usual "single" models without pairs. Simulations show that the disease can spread up to three times more slowly in pair formation models than in the approximated models without pairs.
\end{abstract}

1. Pair Formation and Mixing

Population structure determines the pattern of the spread of a disease. In the AIDS epidemic, for example, there appear to be several risk groups with nonproportional mixing among them. Mixing behavior recently has received a great deal of attention (see for example Jacquez et al. (1988), Sattenspiel and Simon (1988), Hyman and Stanley $(1988,1989)$, Blythe and CastilloChavez (1989)). However, most of the studies of sexually-transmitted diseases (STD's) concentrate on homosexual populations and have not dealt with the heterogeneities introduced by two sexes and pair formation. Although the majority of individuals live in steady partnerships, these models do not follow pairs in the equations. They implicitly assume that the duration of partnerships is zero and that all sexual contacts happen instantaneously. This approximation may be justified in highly sexually active subgroups, but otherwise one has to take into account the fact that pairs of susceptibles are practically immune and that pairs with at least one infected partner do not spread the disease outside the pairs as long as they remain together and do not ve other partners. This can strongly influence the initial phase of an epidemic because the majority of existing pairs consist of susceptible individuals. 
It is therefore important in the modelling of sexually transmitted diseases, as in human demography, to have a mathematical description of the formation and dissolution of pairs. Mixing behavior of individuals produces one constraint on pair formation. Before the formation f a pair there must be an encounter between possible partners. A first approach to pair formation is thus the "encounter-mating" model, in which pair formation involves two steps: the encounter of a possible partner and the decision whether to "mate". The mixing pattern determines the encounter step. The decision to mate is often treated as instantaneous step but this may not be true. Individual preferences are often not easily recognizable at the first encounter (Gimelfarb (1988a,b)). The two terms "mixing" and "preference" are often confused. The terms refer to different phenomena: mixing describes which individuals are met, preference describes which individuals are likely to be chosen (for instance as partners). Whereas mixing between subgroups must be symmetric, i.e. subgroup i mixes as many times with subgroup $j$ as subgroup $j$ mixes with subgroup $i$, preferences need not be symmetric. In models to describe mixing patterns in AIDS, preferences often are not considered.

Until about 1947 all population models considered only one sex. They typically focused on the female population because births are more easily attributable to the mother. But the same models were also applied to the male sex. Kuczynski (1932) calculated the female and male net reproduction numbers for France 1920-3 (the average number of daughters (sons) that will be born to a female (male)) and he found the female rate to be 0.977 and the male rate to be 1.194. One-sex models would therefore predict either a decrease or an increase of the population, depending on the sex. Kuczynski at that time explained these differences in the rates as being due 0 wars.

As a first attempt to overcome the inconsistencies in one-sex population models, A. $\mathrm{H}$. Pollard (1948) attributed artificially the number of male births to females and the number of female births to males. Kendall (1948) suggested some different deterministic approaches to this so-called "two-sex problem". First he considered the simplest one-sex model

$$
x^{\prime}=(\lambda-\mu) x
$$

where $x(t)$ is the number of females at time $t, \lambda$ the birth rate and $\mu$ the death rate. Then he generalized this equation to two sexes:

$$
\begin{aligned}
& x^{\prime}=-\mu x+1 / 2 \Lambda(x, y), \\
& y^{\prime}=-\mu y+1 / 2 \Lambda(x, y),
\end{aligned}
$$

where the term $\Lambda(x, y)$ is symmetric in $x$ and $y$ and describes the births due to males and females. It is easy to see by subtracting one equation from the other, that an initial excess of one sex will disappear in time in this model. Later Kendall (1949) considered a model that explicitly followed single females $\mathrm{x}$, single males $\mathrm{y}$, and couples $\mathrm{p}$ :

$$
\begin{aligned}
x^{\prime} & =-\mu x+(\lambda+\mu) p-\varphi(x, y) \\
y^{\prime} & =-\mu y+(\lambda+\mu) p-\varphi(x, y) \\
p^{\prime} & =-2 \mu p+\varphi(x, y)
\end{aligned}
$$


where the birth and death rates $\lambda$ and $\mu$ are the same for males and females. $\varphi(x, y)$ describes the number of new pairs. Kendall assumed $\varphi(x, y)$ to be $\rho \min (x, y), \rho=$ const. Although this model is quite realistic, it has the disadvantage of assuming that male and female birth and death rates are equal, which is often a poor approximation.

Since 1949 numerous authors have worked on the two sex problem. Keyfitz (1972), Parlett (1972) and J.H.Pollard (1973) designed and discussed models with different mating functions and understood that a realistic mating function is definitely nonlinear. McFarland (1972) and Fredrickson (1973) specified some conditions that had to be satisfied by a mating function:

(i) Definiteness:

In the absence of males and females there should be no

pair formation,

$\varphi(0, y)=\varphi(x, 0)=0$.

(ii) Homogeneity:

If the sex ratio remains constant, the pair formation increases

proportional to the total population size,

$\varphi(\alpha x, \alpha y)=\alpha \varphi(x, y)$ for all $\alpha, x, y \geq 0$.

(iii) Monotonicity:

The pair formation increases if the number of males or females increases $x_{3}$,

$u \geq 0, v \geq 0$ then $\varphi(x+u, y+v) \geq \varphi(x, y)$ for all $x, y \geq 0$.

A consequence of the second condition is that all mating functions can be written in the form

$\varphi(x, y)=x g(y / x)=y h(x / y)$

where $g$ and $h$ are functions for $x, y>0$ of one variable. Hence we can interpret the number of formed pairs per unit time as the number of females times a function of the number of males per female describing the availability of males (or the number of males times another function describing the availability of females). The most common examples in the literature are the minimum function

$$
\varphi(x, y)=\rho \min (x, y),
$$

the geometric mean

$$
\varphi(x, y)=\rho \sqrt{x y}
$$

and the harmonic mean

$$
\varphi(x, y)=2 p x y /(x+y) \text {. }
$$

These demographic models based on Kendall's model assume that the birth rate is linear in the number of pairs. To study the behavior, J. H. Pollard (1973) looked for exponential solutions. Hadeler et al.(1988) confirmed this approach with the theory of homogeneous evolution equations. This theory has also the potential of being applicable to a wider class of population models and epidemiological models (e.g. Nold (1980), Busenberg and van den riessche (1989), Busenberg et. al. (1989)). Hadeler et al. (1988) used this technique to investigate the qualitative behavior of a general two-sex model of the Kendall type. They added a break-up rate for pairs with a general pair-formation law, and showed that if the mortalities of 
males and females do not differ very much there is a globally attractive two-sex exponential solution with constant sex ratio (see also Yellin and Samuelson (1974)). Instead of birth rates depending linearly on the number of pairs, one can also consider a constant recruitment rate $\kappa$ in demographic models:

$$
\begin{aligned}
x^{\prime} & =\kappa-\mu x+(\sigma+\mu) p-\varphi(x, y) \\
y^{\prime} & =\kappa-\mu y+(\sigma+\mu) p-\varphi(x, y) \\
p^{\prime} & =-(\sigma+2 \mu) p+\varphi(x, y)
\end{aligned}
$$

where $\sigma$ is a constant break-up rate, $\mu$ the death rate (independent of sex, for simplicity) and $\varphi$ satisfies the conditions (i)-(iii). In this model exponential solutions do not play an essential role because the equations are not homogenous. There is always a globally stationary solution $(x, y, p)$, where $p$ is determined by the equation

$$
\varphi(\kappa / \mu-p, \kappa / \mu-p)=(\sigma+2 \mu) p
$$

If one assumes that the numbers of both sexes are approximately equal, then the mating functions (8)-(10) are essentially indistinguishable. What then is the value of discrimination among the mating functions? One area where the distinction becomes important is that of agestructured models. Pair formation clearly depends strongly on the ages of the individuals and the umbers in different age classes can be very different.

Several papers about mating models (e.g. Goodman (1967), Fredrickson (1971), Keyfitz (1972), Hoppensteadt (1975), Staroverov (1977), Hadeler (1989a,b), and several others) have treated age structure, which I do not consider further in this paper. Goodman (1953) considers stochastic rather than deterministic models; other papers investigated mating functions in models of population genetics (e.g. Wilson (1973), Wagener (1976) or Karlin (1979)). An application of preferred or assortative mating in one-sex models is presented in Levin and Segel (1982).

2. Two-Sex Models in Sexually Transmitted Diseases

Dietz $(1987,1988)$ and Dietz and Hadeler (1988) presented a two-sex model for diseases spread through sexual contacts among heterosexuals. They assumed that a pair begins with the first sexual contact and that an individual can be member of only one pair at a time. The pulation is divided into eight disjunct classes:

$\mathrm{x}_{0}$ single females, noninfected; 
$\mathrm{x}_{1}$ single females, infected;

yo single males, noninfected;

$\mathrm{y}_{1}$ single males, infected;

po0 pairs, both partners noninfected;

p01 pairs, only male infected;

$\mathrm{p}_{10}$ pairs, only female infected;

$\mathrm{p}_{11}$ pairs, both partners infected.

Individuals are recruited only into the noninfected single classes with a constant rate $\mathrm{k}$. Single females and males are removed with constant death rates $\mu_{0}$ (uninfected) or $\mu_{1}$ (infected), and by forming a pair. Pairs end by breaking up or by death of one partner. The break-up rate is a constant $\sigma$. Furthermore it is assumed that the probability of infection in one sexual contact is a constant $h$ and that the average number of sexual contacts within a pair is $\beta$. Then the model equations read:

$$
\begin{aligned}
& \mathrm{dx}_{0} / \mathrm{dt}=\kappa-\mu_{0} \mathrm{x}_{0}+\left(\mu_{0}+\sigma\right) \mathrm{p}_{00}+\left(\mu_{1}+\sigma\right) \mathrm{p}_{01}-\varphi_{00}-\varphi_{01} \\
& \text { dyo } / \mathrm{dt}=\kappa-\mu_{0} \mathrm{y}_{0}+\left(\mu_{0}+\sigma\right) \mathrm{p}_{00}+\left(\mu_{1}+\sigma\right) \mathrm{p}_{10}-\varphi_{00}-\varphi_{10} \text {; } \\
& \mathrm{dx}_{1} / \mathrm{dt}=-\mu_{1 \mathrm{x}_{1}}+\left(\mu_{0}+\sigma\right) \mathrm{p}_{10}+\left(\mu_{1}+\sigma\right) \mathrm{p}_{11}-\varphi_{10}-\varphi_{11} \\
& \mathrm{dy} 1 / \mathrm{dt}=-\mu_{1} \mathrm{y}_{1}+\left(\mu_{0}+\sigma\right) \mathrm{p}_{01}+\left(\mu_{1}+\sigma\right) \mathrm{p}_{11}-\varphi_{01}-\varphi_{11} \\
& \mathrm{dp} 00 / \mathrm{dt}=-\left(2 \mu_{0}+\sigma\right) \mathrm{p}_{00}+\varphi_{00}
\end{aligned}
$$

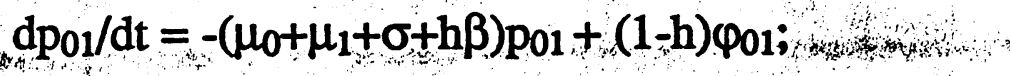

$$
\begin{aligned}
& \mathrm{dp}_{10} / \mathrm{dt}=-\left(\mu_{1}+\mu_{0}+\sigma+\mathrm{h} \beta\right) \mathrm{p}_{10}+(1-\mathrm{h}) \varphi_{10} ; \\
& d p_{11} / d t=-\left(2 \mu_{1}+\sigma\right) p_{11}+h \beta p_{01}+h \beta p_{10}+h \varphi_{01}+h \varphi_{10}+\varphi_{11} \text {. }
\end{aligned}
$$

This model contains the demographic model (11) if there is no infection in the population and I will call it the Dietz/Hadeler or PSI-model (Pair formation - SI - model). The pair formation $\varphi$ is defined by

$$
\varphi_{\mathrm{ij}}\left(\mathrm{x}_{0}, \mathrm{x}_{1}, \mathrm{y}_{0}, \mathrm{y}_{1}\right)=\rho_{\mathrm{ij}} \mathrm{yj}_{\mathrm{i}} /\left(\mathrm{x}_{0}+\mathrm{x}_{1}\right)
$$

which is derived from a model where males of type $j$ meet females of type $i$ with fraction $\mathrm{xi} /\left(\mathrm{x}_{0}+\mathrm{x}_{1}\right)$ and mate with a constant rate $\rho_{\mathrm{ij}}$ without competition (male dominance). Although this function satisfies the conditions (i), (ii), (iii) it has the effect that for very small numbers of females there are a larger number of pairs formed than the total number of females. To avoid this problem the authors assume in their paper that the number of females is greater than or qual to the number of males.

Alternatively, assume that females of type $i$ will encounter males of types 0 and 1 in the proportions $\alpha_{i 0}: \alpha_{i 1}$ and that males of type $j$ will encounter females of types 0 and 1 in the 
proportions $\beta_{0 \mathrm{j}}: \beta_{1 \mathrm{j}}, \alpha_{\mathrm{i} 0}+\alpha_{\mathrm{i} 1}=1$ and $\beta_{0 \mathrm{j}}+\beta_{1 \mathrm{j}}=1$. Assume that $\alpha_{\mathrm{ij}}$ and $\beta_{\mathrm{ij}}$ are density dependent, i.e.

$\alpha_{i j}=\alpha_{i j}\left(y_{0}, y_{1}\right), \quad \beta_{i j}=\beta_{i j}\left(x_{0}, x_{1}\right)$

Furthermore, again let be $\rho_{i j}$ be the probability that a female of type $i$ forms a pair with a male of type $j$, given a meeting. Then

$$
\begin{aligned}
& \varphi_{i j}\left(x_{0}, x_{1}, y_{0}, y_{1}\right)= \\
& 2 \rho_{i j} \alpha_{i j} x_{i} \beta_{i j} y_{j} /\left(\alpha_{i j} x_{i}+\beta_{i j} y_{j}\right) .
\end{aligned}
$$

This function is a generalization of the harmonic mean (10) and of the preferential mating in Levin and Segel (1982). If we assume random mixing,

$$
\alpha_{i j}=y_{j} /\left(y_{0}+y_{1}\right), \quad \beta_{i j}=x_{i} /\left(x_{0}+x_{1}\right),
$$

the mating function (16) simplifies to (see also Hadeler and Ngoma (1989))

$$
\begin{aligned}
& \varphi_{\mathrm{ij}}\left(\mathrm{x}_{0}, \mathrm{x}_{1}, \mathrm{y}_{0}, \mathrm{y}_{1}\right)= \\
& 2 \rho_{\mathrm{ij}} \mathrm{x}_{\mathrm{i}} \mathrm{yj}_{\mathrm{j}} /\left(\mathrm{x}_{0}+\mathrm{x}_{1}+\mathrm{y}_{0}+\mathrm{y}_{1}\right) .
\end{aligned}
$$

If, furthermore, $x_{i}=y_{i}$ for $i=0,1(18)$ is exactly the same function as (14). Dietz and Hadeler (1988) showed that there is always a trivial noninfected stationary solution $\left(\overline{\mathrm{x}}_{0}, \overline{\mathrm{x}}_{0}, 0,0, \overline{\mathrm{p}}_{00}, 0,0,0\right)$. They derived a threshold condition for existence of another stationary solution which is determined by the sign of $\mathrm{D}$, where

$$
\begin{aligned}
D=h \rho_{01}\left[2 \mu_{1}\left(\mu_{1}+\sigma\right)+\sigma\left(\mu_{0}+\sigma+\beta\right)\right] \\
-\mu_{1}\left(2 \mu_{1}+\sigma\right)\left(\rho_{01}+\sigma+\mu_{0}+\mu_{1}+h \beta\right) .
\end{aligned}
$$

Stability analysis is carried out for the special case with symmetric assumptions and no diseaseinduced mortality.

Dietz (1988) compares these results to those of a simplified model which does not explicitly follow pairs. He matches the probability of infection appropriately by adjusting the parameters of the simplified model. Even so, with realistic parameters, the PSI-model reaches its equilibrium about three times more slowly than the approximate model. Also the equilibrium prevalence of the disease is significantly lower in the original model.

The Dietz/Hadeler model presents an initial step towards the development of more general models of disease transmission. A realistic approach to AIDS must take into account more 
complex social and sexual behavior. For instance the use of an average number of sexual contacts within a pair is doubtful. Furthermore, individuals can also get infected through sexual partners other than their "social" partner, such as prostitutes or steady liasons. One should also build both lomosexual pairing and needle sharing by IV drug users into the model. Another idea is to extend the model to variable infectivity over time (see Castillo-Chavez (1989)).

Hadeler and Ngoma (1988) considered a model similar to the PSI-model where they considered vertical transmission. In some diseases the time scales of the recruitment rate and of the demographic process are roughly equal: In this case it may be appropriate to define the recruitment to be linear in the numbers of pairs. Mathematically the model contains Kendall's model (3) if there is no infection. In this case exponential solutions play an important role and the authors use the stability analysis in Hadeler et. al. (1988).

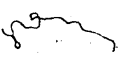

3. Pair Formation Models with Female Prostitutes

Models omitting pair formation and the PSI-model may be seen as two extreme approximations to describe STD's. The former may best describe individuals with many different partners and the latter may best describe individuals who do not have more than one steady partnership at a time. Dietz (1988) showed in simulations that in the PSI-model the virus

spread much more slowly, even if in models without pairs the rate of encounters and the infection rate per encounter are matched appropriately. If individuals have more than one steady partner at a time, i.e. if they have liasons or visit prostitutes, it is expected that the disease will spread faster than in the PSI-model. But how much does this aspect influence the disease? Do pair formation models with more heterogeneities, such as short-term liasons, still show that the disease is spread more slowly and that the prevalence of infectives is lower than in models without pairs? In order to investigate these questions let us introduce two additional classes of female prostitutes, $F_{0}$ and $F_{1}$. Again, 0 means noninfected, 1 infected. For simplicity assume that the interaction of prostitutes and males consists of a single sexual contact with duration zero. Prostitutes become infected through males and transmit the virus into the male population but have no interaction with females (see Fig.2). Let $\psi_{\mathrm{s} d t}$ and $\psi_{\mathrm{c}} \mathrm{dt}$ be the number of sexual contacts per unit time with prostitutes by single men and paired men respectively. One can assume that $\psi \mathrm{s}$ and $\psi \mathrm{c}$ are linear in the male variable,

$$
\psi_{\mathrm{s}}\left(\mathrm{Fi}_{\mathrm{i}, \mathrm{yj}}\right):=\theta_{\mathrm{j}} \mathrm{F}_{\mathrm{i}} /\left(\mathrm{F}_{0}+\mathrm{F}_{1}\right),
$$

$$
\mathrm{sc}_{\mathrm{c}}\left(\mathrm{Fi}_{\mathrm{i}} \mathrm{pkj}\right):=\xi_{\mathrm{pkj}} \mathrm{Fi} /\left(\mathrm{F}_{0}+\mathrm{F}_{1}\right)
$$


Let $\kappa_{F}$ be the constant recruitment rate of noninfected prostitutes and $v_{0}, v_{1}$ the rates at which noninfected and infected prostitutes retire from their business. Let the "social" pair formation be described by the harmonic mean function (16). Since my interest is to look at the possible effects f prostitutes in the dynamics of a STD, we start by random mixing $(17,18)$. The new model reads:

$$
\begin{aligned}
& \mathrm{dx}_{0} / \mathrm{dt}=\kappa-\mu_{0} \mathrm{x}_{0}+\left(\mu_{0}+\sigma\right) \mathrm{p}_{00}+\left(\mu_{1}+\sigma\right) \mathrm{p}_{01}-\varphi_{00}-\varphi_{01} ; \\
& \mathrm{dy} / \mathrm{dt}=\kappa-\mu_{0} \mathrm{y}_{0}+\left(\mu_{0}+\sigma\right) \mathrm{p}_{00}+\left(\mu_{1}+\sigma\right) \mathrm{p}_{10}-\varphi_{00}-\varphi_{10}-\mathrm{h} \psi_{\mathrm{s}}\left(\mathrm{F}_{1}, \mathrm{y}_{0}\right) ; \\
& \mathrm{dx}_{1} / \mathrm{dt}=-\mu_{1} \mathrm{x}_{1}+\left(\mu_{0}+\sigma\right) \mathrm{p}_{10}+\left(\mu_{1}+\sigma\right) \mathrm{p}_{11}-\varphi_{10}-\varphi_{11} \\
& \mathrm{dy}_{1} / \mathrm{dt}=-\mu_{1} \mathrm{y}_{1}+\left(\mu_{0}+\sigma\right) \mathrm{p}_{01}+\left(\mu_{1}+\sigma\right) \mathrm{p}_{11}-\varphi_{01}-\varphi_{11}+\mathrm{h} \psi_{\mathrm{s}}\left(\mathrm{F}_{1}, \mathrm{y}_{0}\right) ; \\
& \mathrm{dp} \mathrm{p}_{00} / \mathrm{dt}=-\left(2 \mu_{0}+\sigma\right) \mathrm{p}_{00}+\varphi_{00}-\mathrm{h} \psi \mathrm{c}\left(\mathrm{F}_{1}, \mathrm{p}_{00}\right) ; \\
& \mathrm{dp}_{01} / \mathrm{dt}=-\left(\mu_{0}+\mu_{1}+\sigma+h \beta\right) \mathrm{p}_{01}+(1-\mathrm{h}) \varphi_{01}+\mathrm{h} \psi \mathrm{c}\left(\mathrm{F}_{1}, \mathrm{p}_{00}\right) ; \\
& \mathrm{dp}_{10} / \mathrm{dt}=-\left(\mu_{1}+\mu_{0}+\sigma+\mathrm{h} \beta\right) \mathrm{p}_{10}+(1-\mathrm{h}) \varphi_{10}-\mathrm{h} \psi_{\mathrm{c}}\left(\mathrm{F}_{1}, \mathrm{p}_{10}\right) \text {; } \\
& \mathrm{dp}_{11} / \mathrm{dt}=-\left(2 \mu_{1}+\sigma\right) \mathrm{p}_{11}+\mathrm{h} \beta \mathrm{p}_{01}+\mathrm{h} \beta \mathrm{p}_{10}+\mathrm{h} \varphi_{01}+\mathrm{h} \varphi_{10}+\varphi_{11} \\
& +\mathrm{h} \psi_{\mathrm{c}}\left(\mathrm{F}_{1}, \mathrm{p}_{10}\right) \\
& \begin{array}{l}
d F_{0} / d t=\kappa_{F}-v_{0} F_{0}-h \psi_{s}\left(F_{0}, y_{1}\right)-h \psi_{c}\left(F_{0}, p_{01}\right)-h \psi_{c}\left(F_{0}, p_{11}\right) \\
d F_{1} / d t=-v_{1} F_{1}+h \psi_{s}\left(F_{0}, y_{1}\right)+h \psi_{c}\left(F_{0}, p_{01}\right)+h \psi_{c}\left(F_{0}, p_{11}\right)
\end{array}
\end{aligned}
$$

will call this model the PSI+P model.

Again there is always a noninfected state $\left(\overline{\mathrm{x}}_{0}, \overline{\mathrm{x}}_{0}, 0,0, \overline{\mathrm{p}}_{00}, 0,0,0, \overline{\mathrm{F}}_{0}, 0\right)$. Assume that the rate pair formation does not depend on the infection

$$
\rho_{00}=\rho_{01}=\rho_{10}=\rho_{11}=\rho,
$$

and furthermore that the pair formation rate with prostitutes is the same for single and coupled men,

$$
\theta=\xi \text {. }
$$

The Jacobian of the system in the noninfected state shows the stability behavior in this infectionfree state. After some cumbersome calculations one gets the threshold condition

$$
\begin{gathered}
D_{1}=v_{0} k^{2}\left[\left(d_{1} \rho^{2}+d_{2} \rho+d_{3}\right)\left(2 \mu_{0}+\sigma\right)+\left(f_{1} \rho^{2}+f_{2} \rho+d_{3}\right) \rho\right] \theta^{2} \\
+\left(l_{1} \rho^{2}+l_{2} \rho+l_{3}\right) \kappa_{F} \mu_{0}\left(\rho+2 \mu_{0}+\sigma_{11}\right)
\end{gathered}
$$

for the noninfected state to be locally asymptotically stable. Here

$$
\begin{aligned}
& d_{1}=-\left[\mu_{1} a(1-h)^{2}+b^{2}\right]<0 \\
& d_{2}=-2 \mu_{1} c_{1}[(1-h) a+b]<0
\end{aligned}
$$




$$
\begin{aligned}
d_{3} & =-\mu_{1} c_{1}^{2}\left(2 \mu_{1}+\sigma\right)<0 \\
f_{1} & =d_{1}+h^{2} a c_{2}<0, \\
f_{2} & =-\left[c_{1}\left\{a\left(2 \mu_{1}(1-h)+h^{2} \beta\right)+2 \mu_{1} b\right\}+h^{2} \beta^{2}(1-h) a\right]<0, \\
\mathrm{~J}_{1} & =v_{1} b\left[(1-h) \mu_{1}\left(2 \mu_{1}+\sigma\right)-h \sigma c_{2}\right], \\
l_{2} & =2 v_{1} \mu_{1}^{2} c_{1}[(1-h) a+b]>0, \\
l_{3} & =v_{1}\left(2 \mu_{1}+\sigma\right) \mu_{1} 2 c_{1} 2>0,
\end{aligned}
$$

and

$$
\begin{aligned}
a & =\mu_{1}+\sigma, b=h\left(\mu_{0}+\sigma+\beta\right)+\mu_{1}, c_{1}=\mu_{0}+\mu_{1}+\sigma+h \beta \\
c_{2} & =\mu_{0}+\mu_{1}+\sigma+\beta
\end{aligned}
$$

If there is no pair formation with prostitutes $(\theta=\xi=0)$, then the threshold condition is identical to that in the Dietz/Hadeler model. Fix $h, \mu_{0}, \mu_{1}, \sigma, \beta, v_{0}, v_{1}, \kappa_{,} \kappa_{F}$ and vary $\rho, \theta$. Then we have the two cases:

Case $1\left(l_{1}<0\right)$

If the average number $\beta$ of sexual contacts within a pair or the infection probability $h$ is "high", then $l_{1}<0$, and we get the following domain of stability:

nair formation rate

ith prostitutes

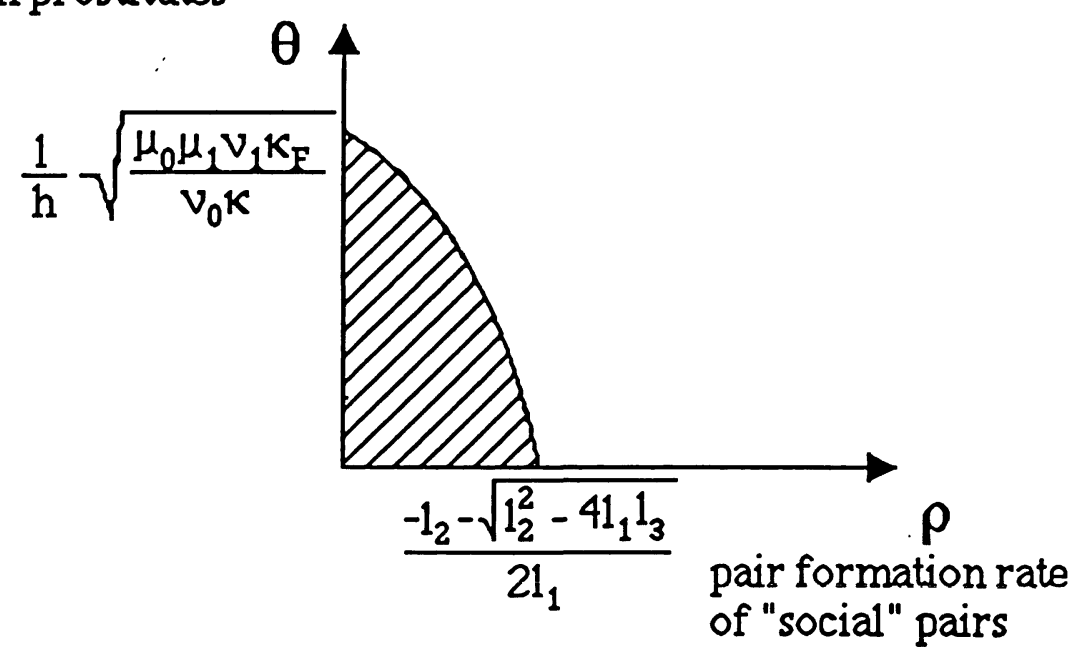

Case $2\left(l_{1}>0\right)$

If $\beta$ or $h$ is "low" then $l_{1}>0$. In that case the "social" pair formation does not play an important role in the spread of the disease. For every $\rho$ there is an interval $\left[0, \theta_{0}\right]$ where the dimase-free equilibrium is still stable. 


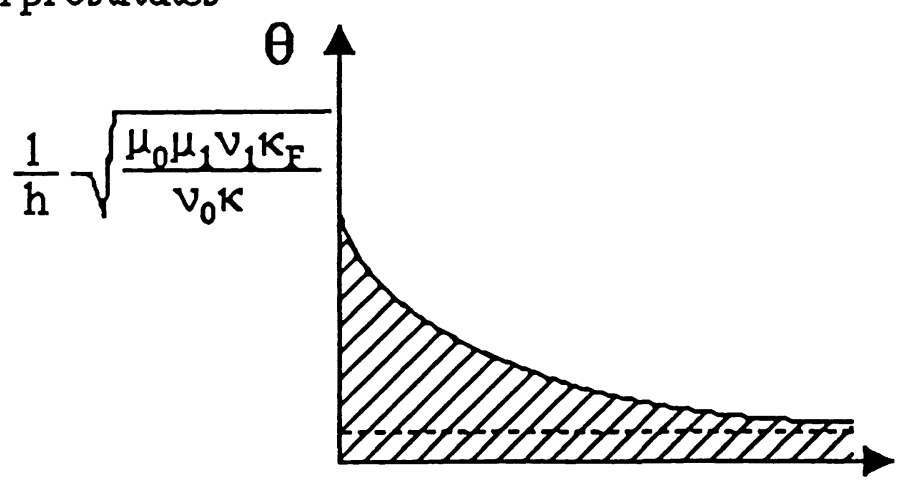

pair formation rate of "social" pairs

In order to understand the effect of considering a class of female prostitutes in pair formation models let us approximate another model. If the break-up rate $\sigma$ tends to infinity in the PSI $+\mathrm{P}$ model we arrive at the following model which neglects pairs:

$$
\begin{aligned}
& \mathrm{dx}_{0} / \mathrm{dt}=\kappa-\mu_{0} \mathrm{x}_{0}-2 \mathrm{kqx}_{0} \mathrm{y}_{1} /\left(\mathrm{x}_{0}+\mathrm{x}_{1}+\mathrm{y}_{0}+\mathrm{y}_{1}\right) \\
& \mathrm{dy} \mathrm{y}_{0} / \mathrm{dt}=\kappa-\mu_{0} \mathrm{y}_{0}-2 \mathrm{kqx}_{1} \mathrm{y}_{0} /\left(\mathrm{x}_{0}+\mathrm{x}_{1}+\mathrm{y}_{0}+\mathrm{y}_{1}\right)-h \theta \mathrm{y}_{0} \mathrm{~F}_{1} /\left(\mathrm{F}_{0}+\mathrm{F}_{1}\right) \\
& \mathrm{dx}_{1} / \mathrm{dt}=-\mu_{1} \mathrm{x}_{1}+2 \mathrm{kqx}_{0} \mathrm{y}_{1} /\left(\mathrm{x}_{0}+\mathrm{x}_{1}+\mathrm{y}_{0}+\mathrm{y}_{1}\right) \\
& \mathrm{y}_{1} / \mathrm{dt}=-\mu_{1} \mathrm{y}_{1}+2 \mathrm{kqx}_{1} \mathrm{y}_{0} /\left(\mathrm{x}_{0}+\mathrm{x}_{1}+\mathrm{y}_{0}+\mathrm{y}_{1}\right)+h \theta \mathrm{y}_{0} \mathrm{~F}_{1} /\left(\mathrm{F}_{0}+\mathrm{F}_{1}\right) \\
& \mathrm{dF}_{0} / \mathrm{dt}=\kappa_{\mathrm{F}}-\mathrm{v}_{0} \mathrm{~F}_{0}-\mathrm{h} \theta \mathrm{y}_{1} \mathrm{~F}_{0} /\left(\mathrm{F}_{0}+\mathrm{F}_{1}\right) \\
& \mathrm{dF}_{1} / \mathrm{dt}=-\mathrm{v}_{1} \mathrm{~F}_{1}+h \theta \mathrm{y}_{1} \mathrm{~F}_{0} /\left(\mathrm{F}_{0}+\mathrm{F}_{1}\right)
\end{aligned}
$$

Here $\mathrm{k}$ is the number of partners per unit time and $\mathrm{q}$ is the probability of getting infected from one partner. This model assumes that all sexual contacts with one partner occur at the same time. $\mathrm{k}$ and $\mathrm{q}$ take the place of the parameters $\rho, \sigma, \beta, \mathrm{h}$ in the original model. For this model the threshold (compare with (25)) is

$$
D_{2}=v_{1} \mu_{0} \kappa_{F}(k q)^{2}+\mu_{1} h^{2} \kappa_{0} \theta^{2}-v_{1} \mu_{0} \mu_{1} 2 \kappa_{F}
$$

To get a sense of the difference between these models with and without pairs, let us assume some realistic values for the parameters. In the noninfected state the total number of (single and coupled) females and males must each be $\kappa / \mu_{0}$. Let $\kappa$ be 200,000 per year and $\mu_{0}=0.02$ per year, so that the number of individuals at risk in the infection-free state is 20 million (Hethcote and Yorke (1984)). Let us further assume that $\kappa_{F}$ is $16,667, v_{0}=0.067$ and $v_{1}=0.125$ per year. Then 
in the infection-free equilibrium we have about 250,000 prostitutes. Let $\rho$ be 4.5 per year, $\theta$ be 1 and $\sigma$ be 0.46 per year, $\mu_{1}=0.1, \mathrm{~h}=0.002$ and $\beta=100$ per year. To match the probability of infection and the pair formation rate in both models, we use the formulas

and

$\mathrm{q}=1-(1-\mathrm{h})^{\mathrm{c}}$, where $\mathrm{c}=1+\beta /\left(\mu_{0}+\sigma\right)$

as in Dietz (1988). We then calculate $k=0.43$ and $q=0.342$ per year.

These parameters yield case 1 in the pair formation model (22). For both models PSI+P and (26) the infection free state is unstable. The models differ in the time to approach the equilibrium. Simulations show that if the noninfected state is unstable, there is always an endemic equilibrium. The model without pairs reaches its equilibrium more than twice as fast as the original model with pairs. The infection prevalence in the endemic equilibrium is significantly lower in the pair formation model (see Fig. 1).

\begin{tabular}{|c|c|c|c|c|}
\hline & \multicolumn{2}{|l|}{ Maximum } & \multicolumn{2}{|c|}{ Equilibrium } \\
\hline & after & \% infected & after & \% infected \\
\hline $\begin{array}{c}\text { Model without } \\
\text { pairs }(26) \\
\theta=0\end{array}$ & $\begin{array}{r}148.5 \\
\text { years }\end{array}$ & $31.4 \%$ & $\begin{array}{l}488.5 \\
\text { years }\end{array}$ & $31.1 \%$ \\
\hline $\begin{array}{c}\text { PSI+P model } \\
\theta=0\end{array}$ & $\begin{array}{l}513.5 \\
\text { years }\end{array}$ & $14.8 \%$ & $\begin{array}{l}689 \\
\text { years }\end{array}$ & $14.8 \%$ \\
\hline $\begin{array}{c}\text { Model without } \\
\text { pairs (26) } \\
\theta=1\end{array}$ & $\begin{array}{l}148 \\
\text { years }\end{array}$ & $32.1 \%$ & $\begin{array}{r}349.5 \\
\text { years }\end{array}$ & $31.5 \%$ \\
\hline $\begin{array}{c}\text { PSI+P model } \\
\theta=1\end{array}$ & $\begin{array}{l}488 \\
\text { years }\end{array}$ & $15.3 \%$ & $\begin{array}{l}665.5 \\
\text { years }\end{array}$ & $15.3 \%$ \\
\hline
\end{tabular}

Fig. 1: Comparison of simulations of the PSI $+\mathrm{P}$ model and model (23). The time to equilibrium is measured when the distance of the trajectory and the equilibrium is for the last time more than 1000 individuals. 


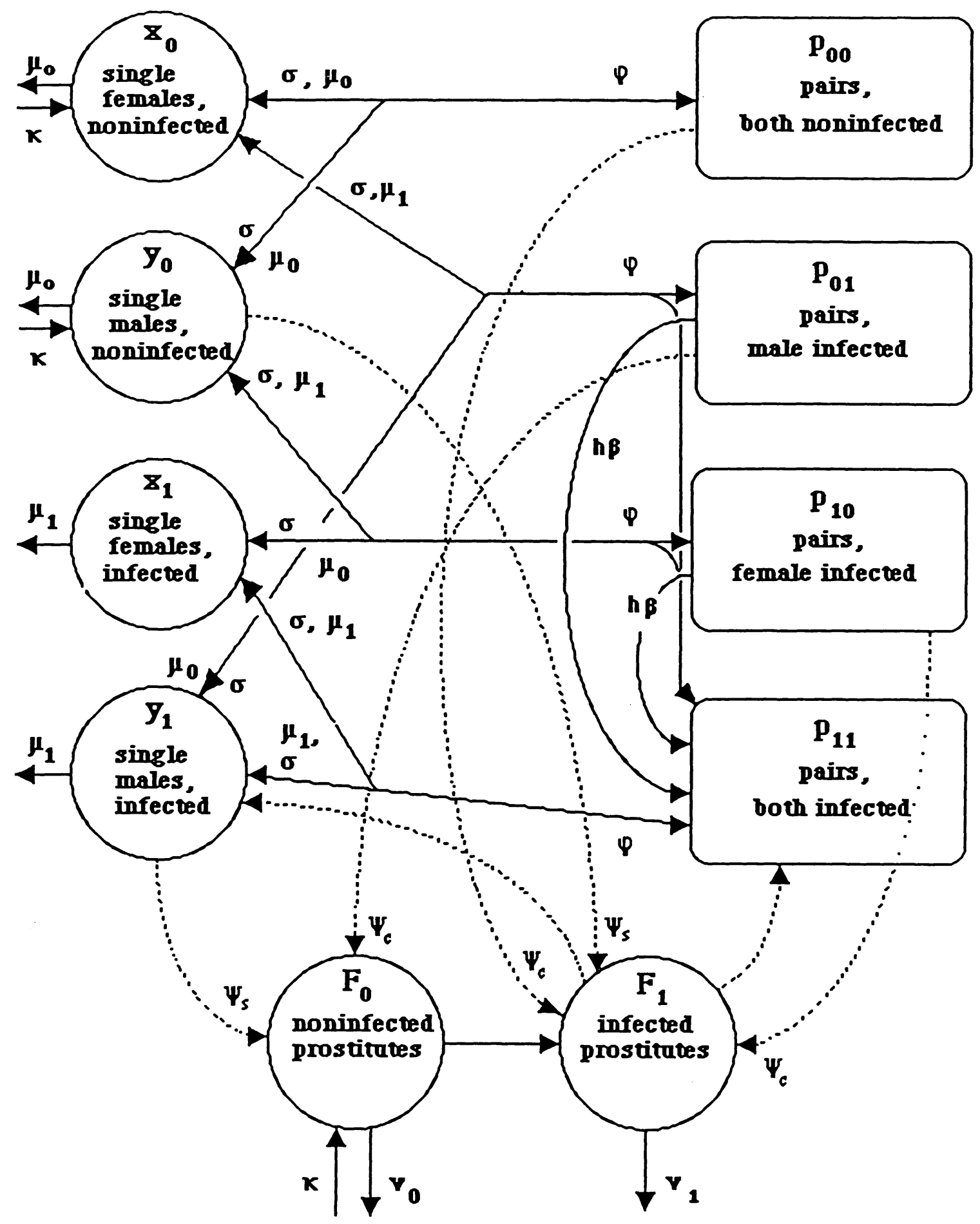

Fig.2: Possible interactions in a two-sex model of heterosexuals with prostitution.

Discussion

A brief overview of pair formation and two-sex models in epidemics has been presented. The model of Dietz and Hadeler has been extended by considering an additional class of female stitutes to look at the effects of female prostitution on the dynamics of pair formation models. It was assumed that prostitutes interact only with the male population. Social structure other than sex still has been ignored. 
Simulations indicate that only high values of $\theta$, the rate at which males visit prostitutes, alter the course of the disease significantly. This result depends strongly on the transmission probability per sexual contact. As in the Dietz/Hadeler model, a comparison to a model not xplicitly considering pairs shows that the prevalence of the disease in the pair formation model is much lower than in the model without pairs, even if prostitution is considered. Whereas in the model without pairs the equilibrium prevalence for certain realistic parameters is about $30 \%$, the pair formation model with female prostitutes shows a prevalence of about $15 \%$. Simulations indicate also that the equilibrium is reached twice as fast in the model without pairs. (see Fig.1).

In reality prostitutes are not a social class "outside of society"; their mixing behavior is much more complex. In models with prostitution there are a lot of uncertainties. Estimates of the number of prostitutes in the United States lie inbetween 80,000 and 800,000 (Castillo-Chavez, personal communication). It is also difficult to get an idea of the magnitude of $\theta$.

Unfortunately pair formation models are very cumbersome to analyze and the number of parameters that have to be estimated is very large. Although simulations cannot replace analytical treatment, one may get some useful insights into the behavior of these models.

Acknowledgements. I would like to thank Carlos Castillo-Chavez and Simon A. Levin whose ideas and suggestions made this work possible. This work was partially supported by the German Exchange Program (DAAD) 315/402/690/9 and by Hatch Project NYC 151-409, USDA, to Carlos Castillo-Chavez. I wish also to thank Fred Adler and Dan Grünbaum for reading and correcting the text and Karl P. Hadeler for valuable comments on the paper, as well as the Center Applied Mathematics, Cornell University, for supporting my work.

\section{REFERENCES}

Blythe, S.P., Castillo-Chavez, C. (1989). Like-with-like preference and sexual mixing models. Math. Biosci. (in press).

Busenberg, S., van den Driessche, P. (1989). Analysis of a disease transmission model in a population with varying size. J. Math. Biol., submitted.

Busenberg, S., Cooke, K.L., Thieme, H. (1989). Investigation of the transmission and persistence of HIV/AIDS in a heterogeneous population. Preprint.

Castillo-Chavez, C. (1989). Review of recent models of HIV/AIDS transmission. In: Levin, S.A., Hallam, T.G., Gross, L.J.,(eds.): Applied Mathematical Ecology. Biomathematics 18, Springer Verlag.

Dietz, K. (1987). Epidemiological models for sexually transmitted infections. Proc. First World Congress Bernoulli Soc., Tashkent 1986, VNU Science Press, Utrecht

Dietz, K. (1988). On the transmission dynamics of HIV. Math. Biosci. 90: 397-414.

Dietz, K., Hadeler, K.P. (1988). Epidemiological models for sexually transmitted diseases. J. Math. Biol. 26, 125.

Fredrickson, A.G. (1971). A mathematical theory of age structure in sexual populations: Random mating and monogamous marriage models. Math. Biosci. 10, 117-143. 
Gimelfarb, A. (1988a). Processes of pair formation leading to assortative mating in biological populations: encounter-mating model. Americ. Natur. 131, No. 6, 865-884.

Gimelfarb, A. (1988b). Processes of pair formation leading to assortative mating in biological populations: dynamic interaction model. Theor. Pop. Biol. 34, 1-23.

Goodman, L.A. (1953). Population growth of the sexes. Biometrics 9, 212- 225.

Goodman, L.A. (1967). On the age-sex composition of the population that would result from given fertility and mortality conditions. Demography 4, 423-441.

Hadeler, K.P. (1989a). Pair formation in age-structured populations. Acta Applic. Math. 14, 91-102.

Hadeler, K.P. (1989b). Modeling AIDS in structured populations. Invited paper of the 47th Biennial Session of the International Statistical Institute, Paris 1989.

Hadeler, K.P., Waldstätter, R., Wörz-Busekros, A. (1988). Models for pair formation in bisexual populations. J. Math. Biol. 26: 635-649.

Hadeler, K.P., Ngoma, K. (1988). Homogeneous models for sexually transmitted diseases. Proc. G. Butler Memorial Conference, Edmonton 1988, Rocky Mtn. Math. Journal to appear.

Hethcote, H.W., Yorke, J.A. (1984). Gonorrhea transmission dynamics and control. Lecture Notes in Biomathematics, No. 56, Springer Verlag, New York.

Hoppensteadt, F.C. (1975). Mathematical theories of populations: demographics, genetics and epidemics. CBMS, vol. 20, SIAM, Philadelphia.

Hyman J.M., Stanley E.A. (1988). A risk base model for the spread of the AIDS virus. Math. Biosci. 90, 415473.

yman J.M., Stanley E.A. (1989). The effect of social mixing patterns on the spread of AIDS. In: C. CastilloChavez, S.A. Levin and C. Shoemaker, eds.), Lecture Notes in Biomathematics, Springer Verlag (in press).

Jacquez, J.A., Simon, C.P., Koopman, J., Sattenspiel, L., Perry, T. (1988). Modeling and analyzing HIV transmission: The effect of contact patterns. Math. Biosci. 92: 119-199.

Karlin, S. (1979). Models of multifactorial inheritance: I. Multivariate formulations and basic convergence results. Theor. Pop. Biol. 15, 308-355.

Kendall, D.G. (1949). Stochastic processes and population growth. Roy. Statist. Soc., Ser B2, 230-264.

Keyfitz, N. (1972). The mathematics of sex and marriage. Proceedings of the Sixth Berkeley Symposium on Mathematical Statistics and Probability. Vol. IV: Biology and Health, pp. 89-108.

Kuczynski, R.R. (1932). Fertility and reproduction, New York: Falcon Press 36-38.

Levin, S.A., Segel, L.A. (1982). Models of the influence of predation on aspect diversity in prey populations. J. Math. Biol. 14: 253-284.

McFarland, D.D. (1972). Comparison of alternative marriage models. In: Greville, T.N.E. (ed.), Population Dynamics, pp.89-106, New York London: Academic Press.

Nold A. (1980). Heterogeneity in disease-transmission modeling. Math. Biosci. 52, 227-240.

lett, B. (1972). Can there be a marriage function ? In: Greville, T.N.E.,(ed.), Population Dynamics, pp.107135, New York London: Academic Press.

Pollard, A.H. (1948). The measurement of reproductivity. J. Inst. Actuaries 74, 288-318. 
Pollard, J.H. (1973). Mathematical models for the growth of human populations, Chap.7: The two sex problem. Cambridge University Press.

Sattenspiel, L., Simon, C.P. (1988). The spread and persistence of infectious diseases in structured populations. Math. Biosci. 90, 341-366.

Staroverov. O.V. (1977). Reproduction of the structure of the population and marriages. (Russian) Ekonomika $i$ matematiceskije metody $13,72-82$.

Wagener D.K. (1976). Preferential mating. Nonrandom mating of a continuous phenotype. Theor. Pop. Biol. 10, 185-204.

Wilson, S.R. (1973). The correlation between relatives under the multifactorial models with assortative mating: I. The multifactorial model with assortative mating. Ann. Human. Genetics 37, 289-304.

Yellin J., Samuelson, P.A. (1974). A dynamical model for human population. Proc. Nat. Acad. Sci. USA 71, No.7, 2813-2817. 